\title{
Atorvastatin Prevents Left Ventricular Remodeling in Spontaneously Hypertensive Rats
}

\author{
Xiao-yan Zhao, ${ }^{1}$ PhD, Ling Li, ${ }^{1}$ MD, Jin-ying Zhang, ${ }^{1}$ PhD, Gang-qiong Liu, ${ }^{1}$ Ya-li Chen, ${ }^{1}$ \\ Pei-ling YANG, ${ }^{1}$ and Rui-yun LiU, ${ }^{1}$
}

\begin{abstract}
SUMMARY
Statins improve left ventricular (LV) remodeling in spontaneously hypertensive rats (SHRs). This study was designed to investigate the effects of atorvastatin administered in the early stage on LV remodeling in SHRs, and to explore the underlying mechanisms.

Sixteen male 8-week-old SHRs were randomized to receive distilled water (SHR-DW) or atorvastatin (SHR-ATV) for 12 weeks. Age-matched male Wistar-Kyoto (WKY) rats gavaged with distilled water served as controls. LV remodeling was evaluated, myocardial CTGF expression levels were detected using Western blotting, and cardiomyocyte apoptosis was detected with the TUNEL method.

Compared with WKY and SHR-DW, atorvastatin treatment significantly decreased systolic blood pressure in SHRs; atorvastatin significantly inhibited LV remodeling, as indicated by the reduced LV weight/body weight ratio (SHR-ATV: $4.0 \pm 0.4$ versus SHR-DW: $4.7 \pm 0.4 \mathrm{mg} / \mathrm{g}, P<0.05$ ), cardiomyocyte diameter (SHR-ATV: $16.2 \pm 2.8$ versus SHR-DW: $19.0 \pm 1.0 \mu \mathrm{m}, P<0.05$ ), and interstitial fibrosis (SHR-ATV: $3.3 \pm 2.1$ versus SHR-DW: $4.5 \pm 1.8 \%, P<$ 0.05). Compared with WKY, myocardial CTGF expression was significantly increased and cardiomyocyte apoptosis decreased in SHRs. Compared with the SHR-DW group, atorvastatin treatment significantly inhibited myocardial CTGF expression (SHR-ATV: $0.69 \pm 0.21$ versus SHR-DW: $1.12 \pm 0.27, P<0.05$ ) and induced cardiomyocyte apoptosis in SHRs (SHR-ATV: $5.2 \pm 0.6$ versus SHR-DW: $1.9 \pm 0.3 \%, P<0.05$ ).

The results indicate that early-stage administration of atorvastatin effectively prevented LV remodeling in SHRs, and that inhibition of myocardial CTGF expression and induction of cardiomyocyte apoptosis may be the underlying mechanisms. (Int Heart J 2010; 51: 426-431)
\end{abstract}

Key words: Atorvastatin, Spontaneously hypertensive rats, Left ventricular remodeling, Connective tissue growth factor

$\mathrm{H}$ ypertension that results in left ventricular (LV) hypertrophy and/or fibrosis can lead to cardiac dysfunction. Persistently increased peripheral resistance and high blood pressure induce a series of compensatory alterations in myocardial structure, including hypertrophy, dilation, and fibrosis. Studies confirmed that, besides blood pressure level, ${ }^{1)}$ many factors (eg, cardiomyocyte apoptosis, unbalanced secretion of cell factors ${ }^{2,3)}$ ) contributed to the development and evolution of LV remodeling in hypertensive models. Investigations into the role of cardiomyocyte apoptosis in the development of LV hypertrophy in animal models produced discrepant results. Some research groups found that cardiomyocyte apoptosis increased in LV hypertrophy and inhibition of apoptosis could alleviate ventricular hypertrophy, ${ }^{4,5)}$ whereas other research teams found contrary results. ${ }^{6}$ Further studies to elucidate the relationship between cardiomyocyte apoptosis and hypertensive LV hypertrophy are therefore required.

Connective tissue growth factor (CTGF), a newly defined cell factor which takes part in regulation of the secretion of extracellular matrix by fibroblasts, has important roles in the fibrosis of various organs, including the liver, kidney, and heart ventricles. ${ }^{7,8)}$ Recent studies have indicated that inhibition of expression of CTGF protein may be a therapeutic target of myocardial fibrosis. ${ }^{9,10)}$ Several studies confirmed the beneficial effects of statins on inhibiting hypertension-induced LV remodeling. Simvastatin mitigates hypertension-induced myocardial fibrosis through down-regulation of myocardial CTGF. ${ }^{11)}$ In rats with pulmonary arterial hypertension, simvastatin prevents and, to some extent, reverses vascular remodeling via down-regulation of CTGF gene expression. ${ }^{12)}$ In cultured rat cardiac fibroblasts and human cardiac fibroblast cells, atorvastatin dose-dependently inhibited transforming growth factor- $\beta$ and angiotensin-II-induced CTGF expression and collagen synthesis. ${ }^{13)}$ In vivo studies revealed that atorvastatin inhibited LV remodeling in spontaneously hypertensive rats (SHRs) through the induction of cardiomyocyte apoptosis; up-regulation of myocardial P27 protein expression; down-regulation of PKD/MEF2D activation; and decreasing blood pressure, serum uric acid concentration, and myocardial angiotensin II concentration. ${ }^{6,14-16)}$ The effects of atorvastatin on myocardial CTGF expression in SHRs are not clear. SHRs develop high blood pressure and LV hypertrophy at an early age, and are a

\footnotetext{
From the ${ }^{1}$ Department of Cardiology, the First Affiliated Hospital, Zhengzhou University, Zhengzhou, China.

This study was supported by the Educational Agency of Henan Province (No. 2009A320025).

Address for correspondence: Ling Li, MD, Department of Cardiology, the First Affiliated Hospital, Zhengzhou University, 1 Jianshe East Road, Zhengzhou 450052,

Received for publication May 26, 2010

Revised and accepted July 30, 2010
} China. 
commonly used model of essential hypertension in humans. Most studies have focused on SHRs aged 12-16 weeks to observe if certain drugs can reverse established LV remodeling; whether atorvastatin administered in the early stage can prevent LV remodeling in SHRs has not been reported yet.

We observed the effects of atorvastatin on LV remodeling, cardiomyocyte apoptosis, and myocardial CTGF protein expression in 8-week-old SHRs to ascertain if atorvastatin administered in the early stage could prevent LV remodeling, and to understand the underlying mechanisms.

\section{MeTHODS}

The investigation conformed with the Guide for the Care and Use of Laboratory Animals published by the US National Institutes of Health (NIH publication number 85-23, revised 1985). Experimental procedures were approved by the Committee of Animal Care and Use of Zhengzhou University (Zhengzhou, China).

Experiments were carried out on 8-week old male SHRs (clean grade, provided by the Laboratory Animal Center, Zhengzhou University) and age-matched male Wistar-Kyoto (WKY) rats. All rats were housed under similar conditions on a 12-hour light/dark cycle with the temperature maintained at $21 \pm 1^{\circ} \mathrm{C}$ and humidity at $55 \pm 5 \%$. The rats had free access to an ordinary diet and water.

Atorvastatin was provided by Pfizer Incorporated (New York, NY). Rabbit polyclonal antibody to CTGF was obtained from Santa Cruz Biotechnology (Santa Cruz, CA, USA), and goat anti-rabbit IgG from Amersham Pharmacia Biotech (Uppsala, Sweden). Horseradish peroxidase (HRP)-conjugated antibody to glyceraldehyde 3-phosphate dehydrogenase (GAPDH) was purchased from Kangchen Biotech (Shanghai, China). The terminal deoxynucleotidyl transferase dUTP nick end labeling (TUNEL) kit was purchased from Roche Diagnostics Limited (Basel, Switzerland). The diaminobenzidine (DAB) kit was from Boster Biological Technology Company Limited (Wuhan, China)

Protocol: SHRs were randomly divided into two groups of 8: spontaneously hypertensive rat-atorvastatin (SHR-ATV) and spontaneously hypertensive rat-distilled water (SHR-DW). For the former, the rats were gavaged with atorvastatin $(50 \mathrm{mg}$ / $\mathrm{kg} \cdot \mathrm{d}$ ) dissolved with $1 \mathrm{~mL}$ of distilled water; for the latter, the rats were gavaged with $1 \mathrm{~mL}$ of distilled water every day. Agematched male WKY rats were gavaged with $1 \mathrm{~mL}$ of distilled water and used as the control.

The treatment was continued for 12 weeks, during which systolic blood pressure (SBP) was measured biweekly in the conscious, lightly restrained rats by tail-cuff plethysmography using a Doppler ultrasonic flowmeter (Abest Electronic Equipment Co., Ltd, Dalian, China) to detect the pulse. SBP was measured an average of 3-5 times for each rat at each measurement. Body weight was recorded every week throughout the study period.

Twelve weeks after the treatment, peripheral arterial blood $(5-6 \mathrm{~mL})$ was collected from the aorta. This was used to detect the concentration of lipids in serum, including total cholesterol (TC), triglycerides (TG), low-density lipoprotein-cholesterol (LDL-C), and high-density lipoprotein-cholesterol (HDL-C). Rats were killed by intra-aortic administration of
$10 \%$ potassium chloride. The left ventricles were isolated, washed with saline, sipped up, and weighed. Some left ventricle specimens were fixed in $10 \%$ neutral buffered paraformaldehyde for histological analysis, while the remaining specimens were snap-frozen in liquid nitrogen for Western blotting. Histological analysis: Some 2-mm-thick transverse sections from the mid-ventricular level were fixed with $10 \%$ neutral buffered paraformaldehyde overnight. They were then processed in paraffin using standard techniques for further histological analysis and determination of apoptosis. Several 5- $\mu \mathrm{m}-$ thick sections were prepared from each specimen, and stained with hematoxylin and eosin (H\&E) for assessment of individual cardiomyocytes as previously described. ${ }^{17,18)}$ One hundred myocardial cells from each rat were selected randomly in a blinded fashion, and the shortest diameters of transversely cut fibers were measured at the level of the nucleus with the aid of an image analyzer. The mean diameter of the 100 cells was calculated.

Several other 5- $\mu$ m-thick sections were prepared from each specimen. Masson's trichrome stain was used to assess the area of fibrotic infiltration in the wall of the left ventricle. ${ }^{19)}$ Green areas showing fibrosis were carefully traced and measured. Papillary muscles and tissue around the vessels were excluded from the measurement of fibrosis. The percentage of fibrotic area in the whole wall of the slices of the left ventricle was obtained by dividing the sum of the fibrotic area of all sections by that of the area of total tissue. For every specimen, the degree of fibrosis was quantified independently by two pathologists in a blinded fashion.

Cardiomyocyte apoptosis was assessed by the TUNEL assay as described. ${ }^{20,21)}$ In brief, nuclear DNA strand breaks were end-labeled with digoxigenin-conjugated dideoxy-UTP by terminal transferase and visualized immunohistochemically with digoxigenin antibody conjugated to alkaline phosphatase. The assay was standardized using adjacent tissue sections treated with DNase I to induce DNA fragmentation as a positive control of apoptosis. Cardiomyocytes with a brown-stained nucleus were identified as positive. The percentage of TUNELpositive cardiomyocytes was calculated in each rat in a transverse left-ventricle tissue section under a microscope (Olympus, Japan) with an ocular grid. A mean of 20 fields for each rat were studied.

Measurement of expression of CTGF protein: Western blotting was performed to analyze the level of expression of CTGF protein in the myocardium. Myocardium tissue was homogenized in lysis buffer. Protein $(60 \mu \mathrm{g})$ was separated by sodium dodecyl sulfate-polyacrylamide gel electrophoresis (SDSPAGE). The protein was then transferred from the gel to polyvinylidene fluoride (PVDF) membranes, which were incubated with anti-CTGF primary antibody (1:300) for 1 hour at room temperature and then at $4{ }^{\circ} \mathrm{C}$ overnight. After washing with TBST buffer, the membranes were incubated with HRP-conjugated goat-anti-rabbit IgG (1:5000) and the signals detected by chemiluminescence. GAPDH was used as an endogenous control to ensure equal loading of the protein.

Statistical analysis: Data are presented as the mean \pm SD and were statistically analyzed by one-way ANOVA with post hoc comparisons using the LSD test. A $P<0.05$ was considered significant. 


\section{Results}

Baseline characteristics: At baseline, there were no significant differences in body weight among the 3 experimental groups. WKY rats had a lower baseline mean heart rate of $359 \pm 8$ beats per minute (bpm) compared with that of the SHRs (SHRDW and SHR-ATV: $425 \pm 14 \mathrm{bpm}$ and $428 \pm 12 \mathrm{bpm}$, respectively, both $P<0.05)$. Significant differences in SBP between the SHR-ATV group and SHR-DW group were not observed (Table I).

Serum lipid levels and blood pressure: After 12-weeks of treatment, the mean serum TC, TG, and LDL-C levels were significantly lower in the SHR-ATV group compared with the WKY

Table I. Baseline Characteristics of Experimental Groups

\begin{tabular}{lcccc}
\hline Group & $n$ & Body weight $(\mathrm{g})$ & Heart rate $(\mathrm{bpm})$ & SBP $(\mathrm{mmHg})$ \\
\hline WKY & 8 & $166 \pm 12$ & $359 \pm 8$ & $125 \pm 12$ \\
SHR-DW & 8 & $160 \pm 10$ & $425 \pm 14^{\mathrm{a}}$ & $168 \pm 12^{\mathrm{a}}$ \\
SHR-ATV & 8 & $157 \pm 10$ & $428 \pm 12^{\mathrm{a}}$ & $170 \pm 12^{\mathrm{a}}$ \\
\hline
\end{tabular}

WKY indicates Wistar-Kyoto rats; SHR-ATV, spontaneously hypertensive rat-atorvastatin; SHR-DW, spontaneously hypertensive rat-distilled water; and SBP, systolic blood pressure. Values are mean \pm SD. ${ }^{a}$ Compared with WKY, $P<0.05$.

Table II. Effects of Atorvastatin on Serum Lipid Levels in Experimental Groups

\begin{tabular}{lccccc}
\hline Group & $n$ & $\begin{array}{c}\text { TC } \\
(\mathrm{mmol} / \mathrm{L})\end{array}$ & $\begin{array}{c}\text { TG } \\
(\mathrm{mmol} / \mathrm{L})\end{array}$ & $\begin{array}{c}\text { LDL-C } \\
(\mathrm{mmol} / \mathrm{L})\end{array}$ & $\begin{array}{c}\text { HDL-C } \\
(\mathrm{mmol} / \mathrm{L})\end{array}$ \\
\hline WKY & 8 & $2.92 \pm 0.26$ & $1.36 \pm 0.09$ & $1.78 \pm 0.18$ & $1.00 \pm 0.09$ \\
SHR-DW & 8 & $2.76 \pm 0.27$ & $1.22 \pm 0.12$ & $1.69 \pm 0.17$ & $0.87 \pm 0.08$ \\
SHR-ATV & 8 & $1.73 \pm 0.18^{\mathrm{ab}}$ & $0.67 \pm 0.09^{\mathrm{ab}}$ & $0.83 \pm 0.07^{\mathrm{ab}}$ & $0.89 \pm 0.09$ \\
\hline
\end{tabular}

TC indicates Total cholesterol; TG, triglycerides; LDL-C, low-density lipoprotein-cholesterol; HDL-C, high-density lipoprotein-cholesterol; WKY, Wistar-Kyoto rats; SHR-DW, spontaneously hypertensive rat-distilled water; and SHR-ATV, spontaneously hypertensive rat-atorvastatin. Values are mean $\pm \mathrm{SD}$. ${ }^{\mathrm{a} C o m p a r e d}$ with WKY of the same age, $P<0.05$; ${ }^{\mathrm{b}}$ Compared with SHR-DW of the same age, $P<0.05$.

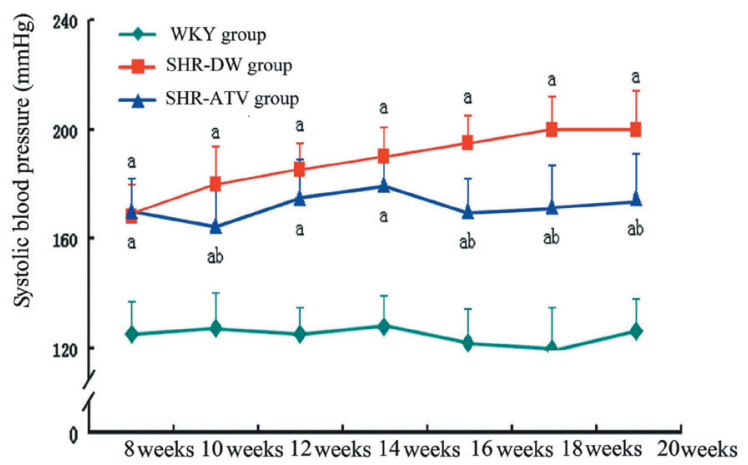

Figure 1. Systolic blood pressure in WKY and SHR rats. WKY indicates Wistar-Kyoto rats; SHR-DW, spontaneously hypertensive rat-distilled water; and SHR-ATV, spontaneously hypertensive rat-atorvastatin. Values are mean $\pm \mathrm{SD}$. ${ }^{\mathrm{a} C}$ Compared with WKY of the same age, $P<0.05 ;{ }^{\mathrm{b}}$ Compared with SHR-DW of the same age, $P<0.05$. and SHR-DW groups, providing direct evidence for the effective delivery of atorvastatin. There was no significant difference in the level of HDL-C in serum among the 3 experimental groups (Table II).

During the 12 week experimental period, SBP was in the normal range in WKY rats and increased along with age in the SHR-DW group. In the SHR-ATV group, SBP was significantly lower than that in the SHR-DW group, though higher than that of the WKY group (Figure 1).

Effects of atorvastatin on $\mathbf{L V}$ remodeling: We evaluated LV remodeling by measuring the ratio of LV weight to body weight, myocardiocyte diameter, and fibrotic infiltration (Table III and Figure 2). Similar body weight was observed in all groups before and after the experiment. At the end of the experiment, the ratio of LV weight to body weight was significantly higher in both SHR groups (SHR-DW and SHR-ATV: $4.7 \pm 0.4 \mathrm{mg} / \mathrm{g}$ and $4.0 \pm 0.4 \mathrm{mg} / \mathrm{g}$, respectively) compared with WKY (3.7 \pm $0.2 \mathrm{mg} / \mathrm{g}$, both $P<0.05$ ), indicating LV hypertrophy in SHRs. Atorvastatin treatment markedly inhibited LV hypertrophy as indicated by a significantly decreased ratio of LV weight to body weight (SHR-ATV versus SHR-DW, $P<0.05$ ).

Photomicrographs of H\&E-stained sections of LV wall confirmed LV hypertrophy in SHRs by revealing an increase in cardiomyocyte diameter (SHR-DW and SHR-ATV: $19.0 \pm$

Table III. Effects of Atorvastatin on Left Ventricular Remodeling

\begin{tabular}{lccccc}
\hline Group & $n$ & $\begin{array}{c}\text { LV weight } \\
(\mathrm{mg})\end{array}$ & $\begin{array}{c}\text { LV weight / } \\
\text { BW }(\mathrm{mg} / \mathrm{g})\end{array}$ & $\begin{array}{c}\text { Cardiomyo- } \\
\text { cyte diameter } \\
(\mu \mathrm{m})\end{array}$ & $\begin{array}{c}\text { Fibrosis infil- } \\
\text { tration }(\%)\end{array}$ \\
\hline WKY & 8 & $1112 \pm 93$ & $3.7 \pm 0.2$ & $14.4 \pm 2.1$ & $2.9 \pm 1.9$ \\
SHR-DW & 8 & $1389 \pm 100^{\mathrm{a}}$ & $4.7 \pm 0.4^{\mathrm{a}}$ & $19.0 \pm 1.0^{\mathrm{a}}$ & $4.5 \pm 1.8^{\mathrm{a}}$ \\
SHR-ATV & 8 & $1257 \pm 113^{\mathrm{ab}}$ & $4.0 \pm 0.4^{\mathrm{ab}}$ & $16.2 \pm 2.8^{\mathrm{ab}}$ & $3.3 \pm 2.1^{\mathrm{ab}}$ \\
\hline
\end{tabular}

WKY indicates Wistar-Kyoto rats; SHR-DW, spontaneously hypertensive rat-distilled water; SHR-ATV, spontaneously hypertensive rat-atorvastatin; and BW, body weight. Values are mean \pm SD. ${ }^{a}$ Compared with WKY of the same age, $P<0.05$; ${ }^{\mathrm{b}}$ Compared with SHR-DW of the same age, $P<$ 0.05 .
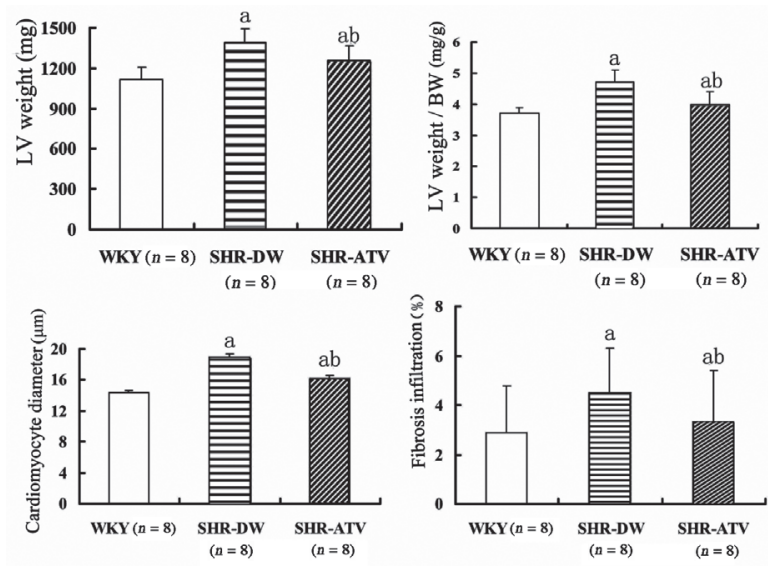

Figure 2. Left ventricular remodeling parameters in WKY and SHR rats. WKY indicates Wistar-Kyoto rats; SHR-DW, spontaneously hypertensive rat-distilled water; SHR-ATV, spontaneously hypertensive rat-atorvastatin; and BW, body weight. Values are mean \pm SD. ${ }^{\mathrm{a}}$ Compared with WKY of the same age, $P<0.05$; ${ }^{\text {b } C o m p a r e d ~ w i t h ~ S H R-D W ~ o f ~ t h e ~ s a m e ~ a g e, ~} P<$ 0.05 . 

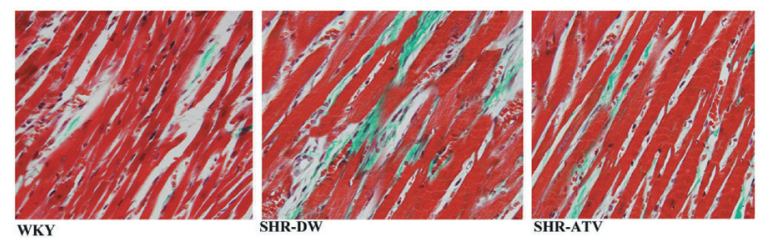

Figure 3. Fibrotic infiltration in the myocardium with Masson's trichrome staining. Original magnification: $\times 400$. Areas stained green represent fibrotic infiltration. Quantitative analysis is shown in Table III and Figure 2.

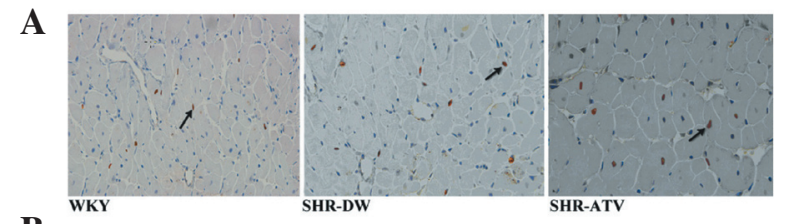

B

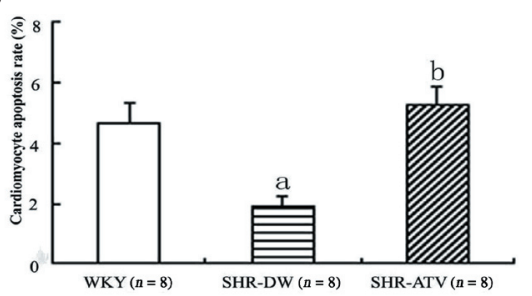

Figure 4. Cardiomyocyte apoptosis detected by the TUNEL method. A: Representative images of the 3 experimental groups. Cardiomyocytes with brown-stained nuclei were defined as "apoptotic" (black arrow). B: Statistical analysis of cardiomyocyte apoptosis. WKY indicates Wistar-Kyoto rats; SHR-DW, spontaneously hypertensive rat- distilled water; and SHRATV, spontaneously hypertensive rat-atorvastatin. Values are mean $\pm \mathrm{SD}$. ${ }^{\mathrm{a} C}$ Compared with WKY of the same age, $P<0.05$; ${ }^{\mathrm{b}}$ Compared with SHRDW of the same age, $P<0.05$.

$1.0 \mu \mathrm{m}, 16.2 \pm 2.8 \mu \mathrm{m}$, respectively) compared with WKY $(14.4 \pm 2.1 \mu \mathrm{m}$, both $P<0.05)$. The increase in cardiomyocyte diameter in the SHR-ATV group was significantly inhibited compared with the SHR-DW group $(P<0.05)$, which confirmed the effects of atorvastatin on preventing LV hypertrophy at the microscopic level.

Photomicrographs of Masson's trichrome-stained sections in the LV wall are shown in Figure 3. Quantitative analysis (Figure 2 and Table III) revealed a significantly higher extent of interstitial fibrosis in the LV wall in the SHR-DW and SHR-ATV groups (SHR-DW and SHR-ATV: $4.5 \pm 1.8 \%, 3.3$ $\pm 2.1 \%$, respectively) compared with WKY rats $(2.9 \pm 1.9 \%$, both $P<0.05)$. Compared with the SHR-DW group, atorvastatin administration significantly alleviated fibrotic deposits in the LV wall (SHR-ATV versus SHR-DW, $P<0.05$ ).

Effects of atorvastatin on cardiomyocyte apoptosis: Cardiomyocytes with brown-stained nuclei were defined as TUNELpositive (Figure 4). Cardiomyocyte apoptosis was evaluated by the ratio of TUNEL-positive cells to the total number of cells (Figure 4B). The apoptosis rate of cardiomyocytes was significantly lower in the SHR-DW group than that in the WKY group $(1.9 \pm 0.3 \%$ versus $4.7 \pm 0.7 \% P<0.05)$. Atorvastatin treatment induced cardiomyocyte apoptosis to a level similar
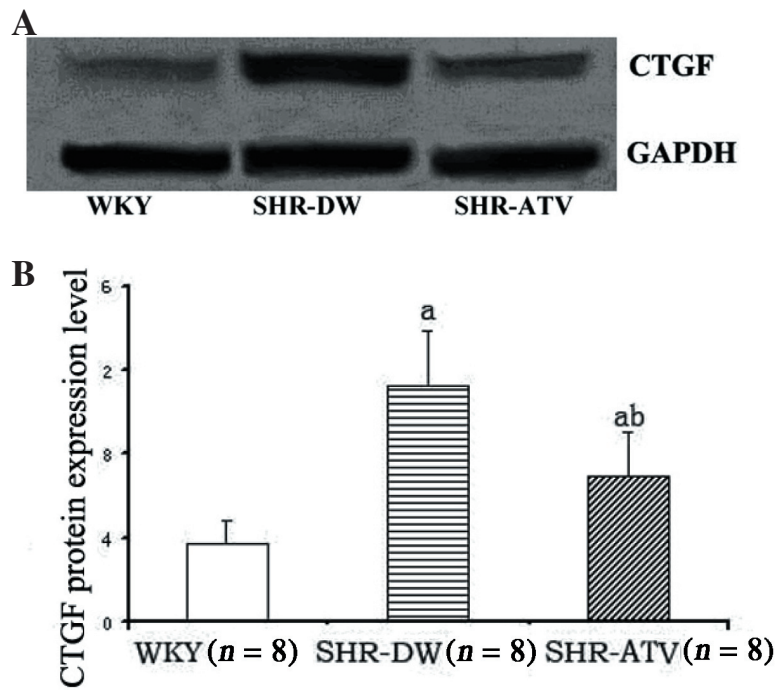

Figure 5. Level of expression of CTGF protein in the myocardium detected by Western blotting. A. Representative images of Western blotting. GAPDH was used as an endogenous control. B. Statistical analysis of the expression of CTGF protein in the myocardium. WKY indicates WistarKyoto rats; SHR-DW, spontaneously hypertensive rat-distilled water; and SHR-ATV, spontaneously hypertensive rat-atorvastatin. Values are mean \pm

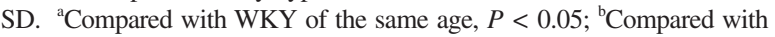
SHR-DW of the same age, $P<0.05$.

to that in WKY rats, which was significantly higher than that in the SHR-DW group $(5.2 \pm 0.6 \%$ versus $1.9 \pm 0.3 \%, P<$ $0.05)$.

Effects of atorvastatin on the level of expression of CTGF protein in the myocardium: Myocardial CTGF protein expression level was significantly up-regulated in the SHR-DW group compared with the WKY group $(1.12 \pm 0.27$ versus $0.37 \pm$ $0.11, P<0.05$ ) (Figure 5). Atorvastatin administration markedly inhibited myocardial CTGF protein expression in SHRs (SHR-ATV versus SHR-DW: $0.69 \pm 0.21$ versus $1.12 \pm 0.27$, $P<0.05)$

\section{Discussion}

Rearrangement of the original structure of the myocardium, accompanied with alterations of the shape of the left ventricle, and interstitial fibrosis, are characteristics of LV remodeling. The effects of statins on LV remodeling and the underlying mechanisms have attracted much attention in recent years. SHRs, a commonly used model of primary hypertension that shows high blood pressure from 6 weeks of age, develop LV hypertrophy accompanied with interstitial fibrosis from 10 weeks of age. In the present study, we administered atorvastatin to 8 week-old SHRs, when blood pressure is increased and LV remodeling has not been established. The randomized placebo-controlled study showed that administration of atorvastatin to SHRs, early and before establishment of LV remodeling, prevented development of cardiac hypertrophy and interstitial fibrosis. Prevention of cardiac hypertrophy was demonstrated at the organ and cell levels. The beneficial effects of atorvastatin were associated with a reduced level of expression of myo- 
cardial CTGF protein, more TUNEL-positive cells, and reduced SBP. These findings indicate the potential utility of atorvastatin in preventing evolving cardiac hypertrophy and fibrosis infiltration in hypertension.

It has been confirmed that apoptosis is a ubiquitous phenomenon in the physiology and pathophysiology of the cardiovascular system. ${ }^{22,23)}$ Hamet, et $a l^{24)}$ were the first to report apoptosis in all the main target organs of hypertension; they found that the apoptosis rate was correlated with the severity of ventricular hypertrophy. Further studies found that cardiomyocyte apoptosis presented dynamic alterations in the course of LV remodeling, ${ }^{25,26)}$ but the exact part it plays in ventricular hypertrophy is incompletely understood. Some researchers reported that a reduction in cardiomyocyte apoptosis has an important role in the development of ventricular remodeling in SHRs, and that induction of apoptosis alleviates ventricular hypertrophy. ${ }^{26,27)}$ In the present study, we chose 8-week-old SHRs (when LV hypertrophy has not yet developed) to evaluate if atorvastatin can prevent hypertension-induced LV remodeling. The apoptosis rate in myocardiocytes in the SHR-DW group was reduced, whereas 12-week treatment with atorvastatin induced myocardiocyte apoptosis accompanied by alleviated LV remodeling. This indicated that in SHRs aged 8-20 weeks, reduced apoptosis in myocardiocytes was involved in the development of LV remodeling, and that the effects of atorvastatin on apoptosis may be applicable only to SHRs of this age. Studies on myocardiocyte apoptosis and the effects of atorvastatin on this parameter in SHRs older than 20 weeks should be carried out.

CTGF (or fragments thereof) has been postulated to be involved in conditions in which there is an overgrowth of connective tissue cells (eg, systemic sclerosis, cancer, fibrotic conditions, atherosclerosis) as well as in wound healing. The synthesis and secretion of CTGF are selectively induced by TGF- $\beta$. It has been shown that the synthesis and action of CTGF are essential for TGF- $\beta$ to stimulate fibroblast growth. Studies showed that angiotensin II increased CTGF protein expression in adventitia fibroblasts via the AT1 receptor, ${ }^{28)}$ and that the effects of an angiotensin II receptor antagonist on alleviating myocardial fibrosis were related to inhibiting CTGF expression, ${ }^{29)}$ which is up-regulated in hypertensive rats. Many studies have confirmed that statins have beneficial effects on LV remodeling in SHRs. In cultured vascular smooth muscle cells, atorvastatin and simvastatin inhibited angiotensin II-induced CTGF production. ${ }^{12)}$ In a renovascular hypertensive rat model, simvastatin inhibited myocardial CTGF expression and fibrosis infiltration. ${ }^{11)}$ The effects of atorvastatin on myocardial CTGF expression in SHRs have rarely been reported. In the present study, treatment with atorvastatin for 12 weeks in SHRs significantly reduced myocardial CTGF expression, and was accompanied by markedly alleviated fibrosis infiltration. This indicated that inhibiting myocardial CTGF expression to inhibit myocardial fibrosis may be one of the mechanisms of the beneficial effects of atorvastatin on LV remodeling in SHRs.

Hypertension induced by increased mechanical tension is one of the important factors causing LV hypertrophy, and lowering of blood pressure could effectively inhibit LV remodeling. Several research groups recently reported the effects of statins on blood pressure, ${ }^{30,31)}$ and the results indicated that statins showed blood pressure-lowering effects (though the exact magnitude of this action needs to be clarified). In the present study, atorvastatin administration significantly reduced SBP in SHRs, and was accompanied by improved LV hypertrophy and myocardial fibrosis infiltration. The results indicated that the blood pressure-lowering effects of atorvastatin may be associated with its beneficial effects on LV remodeling. Meliorating vascular endothelial function and reduced levels of circulating endothelin-1 and angiotensin II receptors have been proposed to be involved in the effects of statins on modulating blood pressure. $^{30)}$

Study limitations: Cardiomyocyte apoptosis and the level of myocardial CTGF expression, and the effects of atorvastatin on these parameters, should be observed dynamically. The beneficial effects of atorvastatin on myocardial fibrosis related to augmenting collagen degradation or decreasing collagen synthesis need further investigation.

Conclusion: It has been confirmed that atorvastatin can improve or partly reverse LV remodeling in SHRs. The results of the present study indicate that atorvastatin administered at an early stage can prevent LV hypertrophy and myocardial fibrosis. Induction of cardiomyocyte apoptosis, inhibition of CTGF expression in the myocardium, and reduction in SBP may be the underlying mechanisms of this phenomenon.

\section{ACKNOWLEDGMENT}

We thank Pfizer Inc. (USA) for providing the atorvastatin.

\section{REFERENCES}

1. Ishikawa Y. High blood pressure and cardiac hypertrophy. Nippon Rinsho 2004; 62: 342-6. (Review) (Japanese)

2. Sugden PH, Clerk A. Cellular mechanisms of cardiac hypertrophy. J Mol Med 1998; 76: 725-46. (Review)

3. Buemi M, Corica F, Marino D, et al. Cardiovascular remodeling, apoptosis, and drugs. Am J Hypertens 2000; 13: 450-4. (Review)

4. Louhelainen M, Vahtola E, Kaheinen P, et al. Effects of levosimendan on cardiac remodeling and cardiomyocyte apoptosis in hypertensive Dahl/Rapp rats. Br J Pharmacol 2007; 150: 851-61.

5. Sakairi A, Ishida J, Honjo K, et al. Angiotensin type 1 receptor blockade prevents cardiac remodeling in mice with pregnancy-associated hypertension. Hypertens Res 2008; 31: 2165-75.

6. Kang L, Ge CJ, Hu SJ. Beneficial effect of atorvastatin on left ventricular remodeling in spontaneously hypertensive rats. Pharmacology 2007; 80: 120-6.

7. Li G, Li D, Xie Q, Shi Y, Jiang S, Jin Y. RNA interfering connective tissue growth factor prevents rat hepatic stellate cell activation and extracellular matrix production. J Gene Med 2008; 10: 103947.

8. Riser BL, Najmabadi F, Perbal B, et al. CCN3 (NOV) is a negative regulator of CCN2 (CTGF) and a novel endogenous inhibitor of the fibrotic pathway in an in vitro model of renal disease. Am J Pathol 2009; 174: 1725-34

9. Duisters RF, Tijsen AJ, Schroen B, et al. miR-133 and miR-30 regulate connective tissue growth factor: implications for a role of microRNAs in myocardial matrix remodeling. Circ Res 2009; 104: 170-8.

10. Wang B, Haldar SM, Lu Y, et al. The Kruppel-like factor KLF15 inhibits connective tissue growth factor (CTGF) expression in cardiac fibroblasts. J Mol Cell Cardiol 2008; 45: 193-7.

11. Iwanciw D, Rehm M, Porst M, Goppelt-Struebe M. Induction of connective tissue growth factor by angiotensin II: integration of signaling pathways. Arterioscler Thromb Vasc Biol 2003; 23: 
$1782-7$.

12. Rupérez M, Rodrigues-Díez R, Blanco-Colio LM, et al. HMG$\mathrm{CoA}$ reductase inhibitors decrease angiotensin II-induced vascular fibrosis: role of RhoA/ROCK and MAPK pathways. Hypertension 2007; 50: 377-83.

13. Martin J, Denver R, Bailey M, Krum H. In vitro inhibitory effects of atorvastatin on cardiac fibroblasts: implications for ventricular remodelling. Clin Exp Pharmacol Physiol 2005; 32: 697-701.

14. Geng J, Zhao Z, Kang W, Wang W, Zhang Y, Zhiming GE. Atorvastatin reverses cardiac remodeling possibly through regulation of protein kinase $\mathrm{D} /$ myocyte enhancer factor $2 \mathrm{D}$ activation in spontaneously hypertensive rats. Pharmacol Res 2010; 61: 40-7.

15. Ge CJ, Lu SZ, Chen YD, Wu XF, Hu SJ, Ji Y. Synergistic effect of amlodipine and atorvastatin on blood pressure, left ventricular remodeling, and C-reactive protein in hypertensive patients with primary hypercholesterolemia. Heart Vessels 2008; 23: 91-5.

16. Kang L, Hu SJ. Effect of atorvastatin on left ventricular remodeling in spontaneously hypertensive rats. Zhejiang Da Xue Xue Bao Yi Xue Ban 2007; 36: 54-60. (Chinese)

17. Zhao XY, Hu SJ, Li J, et al. rAAV-mediated angiogenin gene transfer induces angiogenesis and modifies left ventricular remodeling in rats with myocardial infarction. J Mol Med 2006; 84 $1033-46$

18. Czarnowska E, Gajerska-Dzieciatkowska M, Kuśmierski K, et al. Expression of SDF-1-CXCR4 axis and an anti-remodelling effectiveness of foetal-liver stem cell transplantation in the infarcted rat heart. J Physiol Pharmacol 2007; 58: 729-44.

19. Lal A, Veinot JP, Ganten D, Leenen FH. Prevention of cardiac remodeling after myocardial infarction in transgenic rats deficient in brain angiotensinogen. J Mol Cell Cardiol 2005; 39: 521-9.

20. Kytö V, Saraste A, Saukko P, et al. Apoptotic cardiomyocyte death in fatal myocarditis. Am J Cardiol 2004; 94: 746-50.

21. Poelmann RE, Gittenberger-de Groot AC. Apoptosis as an instrument in cardiovascular development. Birth Defects Res C Embryo
Today 2005; 75: 305-13. (Review)

22. Anselmi A, Lotrionte M, Biondi-Zoccai GG, Galiuto L, Abbate A. Left ventricular hypertrophy, apoptosis, and progression to heart failure in severe aortic stenosis. Eur Heart J 2005; 26: 2747.

23. Hamet P, Richard L, Dam TV, et al. Apoptosis in target organs of hypertension. Hypertension 1995; 26: 642-8.

24. Hamet P, Moreau P, Dam TV, et al. The time window of apoptosis: a new component therapeutic strategy for cardiovascular remodeling. J Hypertens Suppl 1996; 14: S65-70. (Review)

25. Li FQ, Chen YZ. Changes and significance of cardiac myocyte apoptosis in spontaneously hypertensive rat. Chin J Cardiol 1999; 27: 459-63.

26. Ikeda S, Hamada M, Qu P, et al. Relationship between cardiomyocyte cell death and cardiac function during hypertensive cardiac remodeling in Dahl rats. Clin Sci (Lond) 2002; 102: 329-35.

27. Ogata Y, Takahashi M, Takeuchi K, et al. Fluvastatin induces apoptosis in rat neonatal cardiac myocytes: a possible mechanism of statin-attenuated cardiac hypertrophy. J Cardiovasc Pharmacol 2002; 40: 907-15.

28. Che ZQ, Gao PJ, Shen WL, Fan CL, Liu JJ, Zhu DL. Angiotensin II-stimulated collagen synthesis in aortic adventitial fibroblasts is mediated by connective tissue growth factor. Hypertens Res 2008; 31: $1233-40$

29. Bai YP, Liu CH, Yang L, Liu WJ. Correlation between connective tissue growth factor and fibrosis of myocardium in spontaneously hypertensive rats and intervention with irbesartan. Chinese Journal of Geriatric Heart Brain and Vessel Diseases 2006; 8: 47-50.

30. Sarafidis PA, Kanaki AI, Lasaridis AN. Effects of statins on blood pressure: a review of the experimental and clinical evidence. Curr Vasc Pharmacol 2007; 5: 155-61. (Review)

31. Golomb BA, Dimsdale JE, White HL, Ritchie JB, Criqui MH. Reduction in blood pressure with statins: results from the UCSD Statin Study, a randomized trial. Arch Intern Med 2008; 168: 721-7. 